\title{
Motor-Vehicle crashes during pregnancy: a retrospective cohort study
}

\author{
Harold B. Weiss ${ }^{1,2}$, Erin K. Sauber-Schatz ${ }^{1,3}$, Amy H. Herring ${ }^{4}$ \\ ${ }^{1}$ University of Pittsburgh, Department of Neurological Surgery, Center for Injury Research and Control, Pittsburgh, USA (At the time \\ of study); \\ ${ }^{2}$ University of Otago, Department of Preventive and Social Medicine, Injury Prevention Research Unit, Dunedin, New Zealand \\ (Currently); \\ ${ }^{3}$ Centers for Disease Control and Prevention, National Center for Injury Research and Control, Division of Unintentional Injury, \\ Atlanta, USA (Currently); \\ ${ }^{4}$ Department of Biostatistics and Carolina Population Center, The University of North Carolina, Chapel Hill, USA. \\ Email: hw@injurycontrol.com
}

Received 8 October 2011; revised 17 November 2011; accepted 2 December 2011.

\begin{abstract}
Background: The purpose of this study was to assess the risk of motor-vehicle pregnant driver crashes in Pennsylvania using vital statistics linked to police and ambulance reports. This was supplemented with a review of national age and sex specific crash and fertility data to put this risk into perspective and rank the likelihood for pregnancy-related crashes in other states. Methods: Motor vehicle police crash reports from the Pennsylvania Department of Transportation were probabilistically linked to four years of birth and fetal death data and five years of infant death records and ambulance reports. State specific motor-vehicle traffic injury rates (fatal and non-fatal) were compared to birth rates, by age, for women ages 15 - 34. Results: 5929 (1.1\%) of the women with a birth or fetal death linked to a police reported motor vehicle driver crash during pregnancy. One-third $(32.5 \%)$ of these crashes resulted in minor maternal injuries and $7.5 \%$ resulted in moderate to fatal maternal injuries. Crashes were evenly distributed across gestational ages. Young drivers (20 - 24) were at highest risk. Police reported nonbelt use was $10 \%$. Conclusions: This study quantifies the risk of motor vehicle crashes during pregnancy in Pennsylvania and offers a perspective on potential variations in other states. Pregnancy related crashes occur at a higher rate than infant related crashes with a concomitant threat to the fetus and newborn not usually tracked within current crash data systems.
\end{abstract}

Keywords: Maternal Injury; Motor Vehicle Crash

\section{INTRODUCTION}

As the infectious scourges of childbirth during the early part of the 20th century yielded to advances in modern health care, women of reproductive age began to take to the road in motor vehicles as never before. The resulting changes in pregnancy-associated mortality and morbiddity have led to motor-vehicle related injuries becoming the leading cause of maternal death during pregnancy $[1,2]$, the leading cause of hospitalized maternal trauma during pregnancy [3-5], for the fetus the leading cause of traumatic fetal injury mortality [6], and in 2007 the leading cause of childhood injury death for children aged 3 and older [7]. It has been shown that there are more fetal deaths due to maternal crashes than there are infant deaths in motor vehicle crashes [2].

The ubiquity of motor-vehicle travel by pregnant women, in combination with their crash risk, has led to reports from national sample surveys that about 2\% - 3\% of all live births in the U.S. are annually exposed inutero to a police-reported crash [8]. This means about 100,000 children (rate $=26 / 1000$ person-years) in the United States (US) may be annually exposed in-utero to the direct and indirect impact of a motor-vehicle crash. For comparison, NHTSA reports that there were an estimated 58,606 infants in crashes in 2008 [9]. Fetal motor-vehicle (MV) crashes have probably increased substantially over the last two decades primarily because more women are driving and driving more miles [10].

Other health sciences that study human exposure to toxic, infectious, and radiologic agents pay a great deal of attention to in-utero exposure, a known critical period in human development. With few exceptions, however, the injury field has not. This paper uses a probabilistic matching study to characterize the number of pregnancy re- 
lated crashes in one state. It is supplemented with a review of national age and sex specific crash and fertility data to rank the likelihood for pregnancy-related crashes in other states.

\section{METHODS}

We used a retrospective cohort design approved by the University of Pittsburgh Institutional Review Board. Identifying pregnant drivers in crashes was achieved by probabilistically linking records from several different databases (police crash reports, births, fetal deaths and ambulance run reports) using common variables to determine the probability that two records referred to the same person and/or event. To identify cases for which the crash occurred during pregnancy, the following case selection was applied: If the gestational age (in weeks) at birth or fetal death was available, we looked back in time for a crash event within the gestational age plus one week. If only the date of last menses was noted and the date of last menses occurred after the woman's reported crash the case was excluded. The few records without any indication of gestational age or last menses were excluded.

Our aim was to link four years of Pennsylvania birth and fetal death data (2002-2005) with drivers in crashes along with any available matching ambulance run reports. Ambulance run reports allowed for more specific crash severity and other injury information than police reports. Only drivers were included in the study because Pennsylvania crash data, like most states, only contains the names of drivers, making linkage to passengers problematic. However, previous work has shown that about $70 \%$ of pregnant women in crashes are the driver [8]. In order to avoid misclassification of crash exposure, for infants born in 2002 or infant deaths of infants born in 2005, crash data from 2001 and infant death data for 2006 were linked to 2002-2005 birth, crash, fetal and infant death data.

Linkage was performed using Automatch software (2008, Strategic Matching, Inc). In order to keep linkage quality high, a priori criteria were established for selecting matched cases. Using the software, we aimed for $90 \%$ specificity and $95 \%$ sensitivity for the crash to the birth and fetal death linkage and 90\% specificity and 90\% sensitivity for the crash to EMS linkage. By administrative fiat from the Department of Transportation we were not allowed to use the existing social security numbers to link driver records with birth data. However, names (sometimes only initials), maternal birth dates and addresses were usually available for the crash driver to vital statistics linkage and therefore adequate linkage could be accomplished. The analytical team only received de-identified data as an honest broker (Strategic Matching, Inc.) performed all data linkage. For rate calculations, in the rare event that more than one crash occurred during the same pregnancy only the data for the most severe crash was included.

\section{RESULTS}

We identified 5929 registered births and fetal deaths from 2002-2004 linking to a female driver crash report. Among these linked cases, about a third (31.7\%) were linked to an EMS run report $(n=1878)$. Of the total birth outcomes, 5885 were live births and 44 were fetal deaths. With 562,993 birth and fetal deaths reported for 2002-2004 this results in a maternal crash rate (crashes among all reported pregnancies) of $1.1 \%$. A small number of mothers were identified with multiple crashes; $1.5 \%(n=91)$ had two crashes and two had three crashes during the same pregnancy. For all pregnancy-related crashes, the mean age of the mother at the time of the infants birth was 26.9 years (range 14 to 51). Figure 1

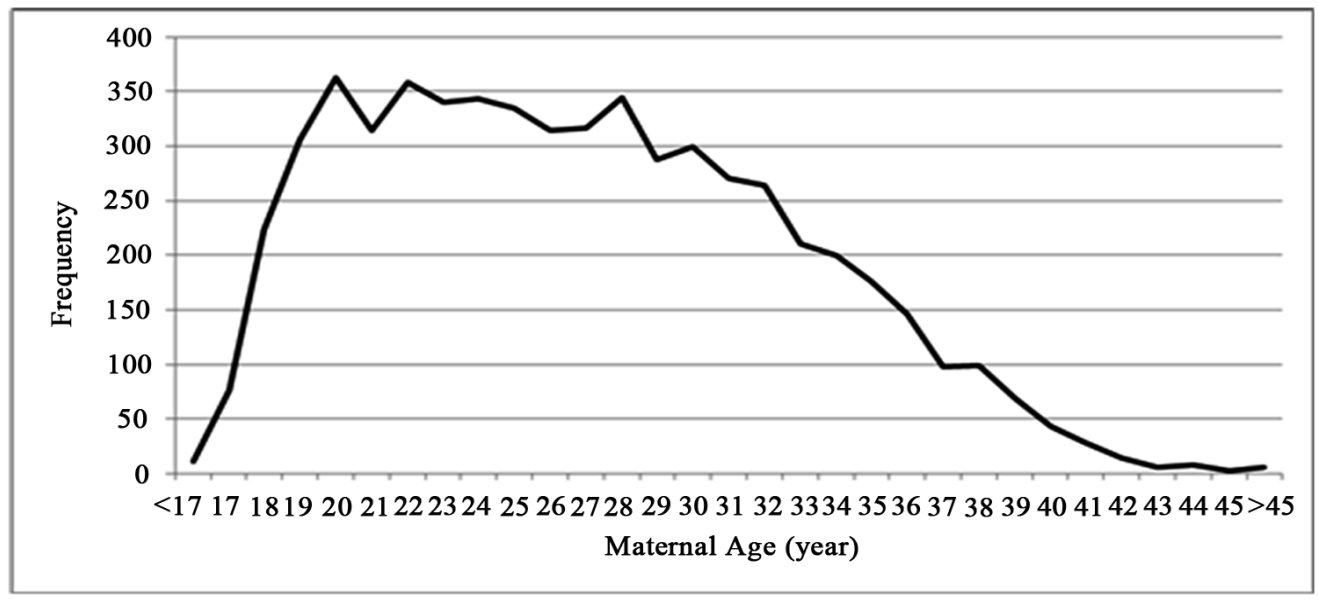

Figure 1. Mothers age at birth or fetal death in pregnancy related driver crashes, Pennsylvania, 2002 to 2005. 


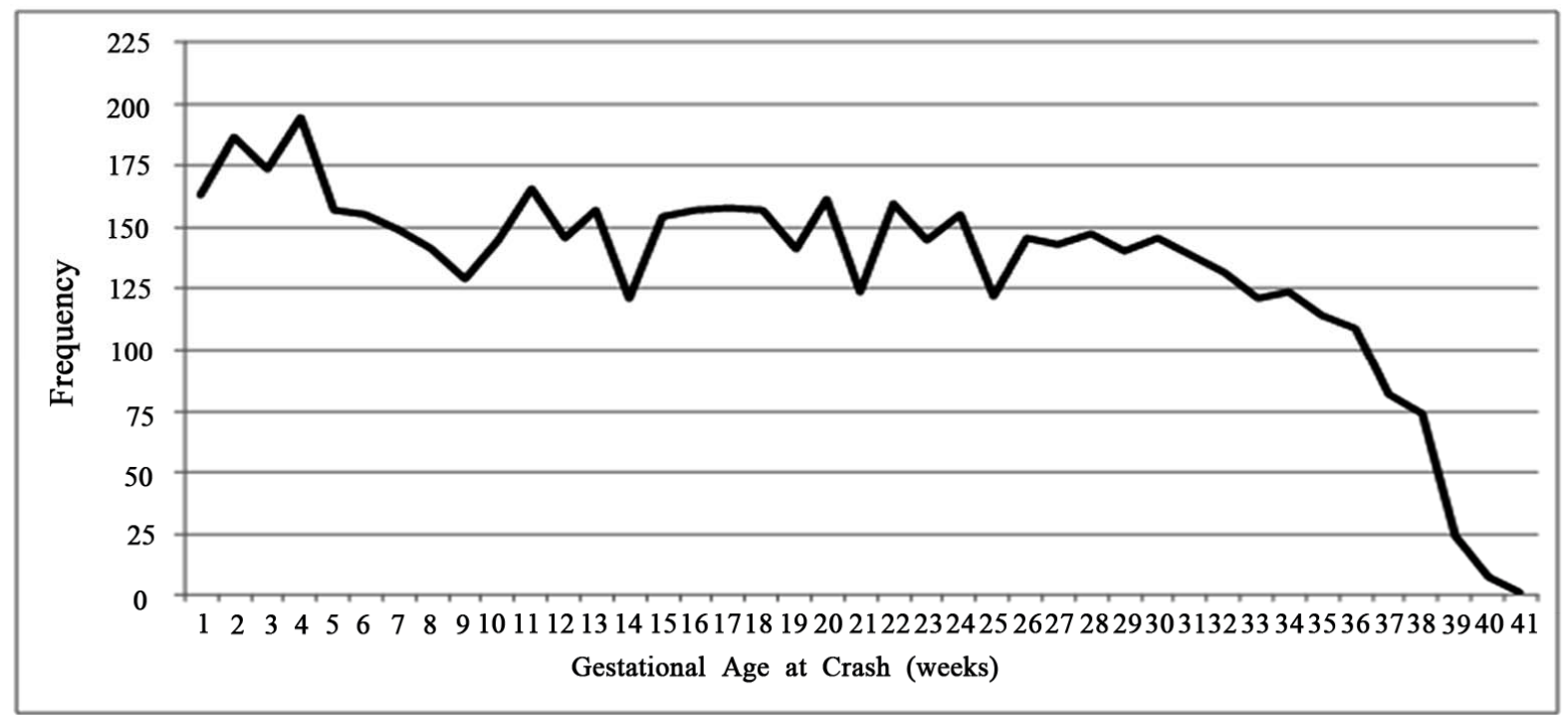

Figure 2. Gestational age in weeks of fetus at time of maternal crash, Pennsylvania, 2002 to 2005. ${ }^{\text {a }}$ (a. Excludes 130 cases with missing gestational age).

shows the frequency of all the first crashes by maternal age. The age-specific rate of maternal crashes per 1000 live births was highest among mothers ages 20 - 24 at 1.34 crashes per 1000 live births. Other rates per 1000 live births were as follows: 15 years -19 years $=1.19$, 25 years -29 years $=1.03$, 30 years -34 years $=0.82,35$ years -39 years $=0.78$, and 40 years -44 years $=0.67$.

Police reported injury severity indicated that $48.3 \%$ of the pregnant women drivers in a crash were not injured, $32.5 \%$ had a minor injury, $7.5 \%$ had a moderate or major injury and one fatality was reported. Crashes were more likely to occur on a weekday than a weekend. Weekday crashes ranged from $15.0 \%$ on Monday to $17.8 \%$ on Friday with Saturday and Sunday crashes accounting for $10.7 \%$ and $8.5 \%$, respectively. Collision angles were mostly either frontal (35.8\%) or a rear-end collision (34.2\%). Most maternal crashes (82.1\%) had no contributing environmental crash factors (such as rain or snow) reported, but $7.8 \%$ indicated slippery conditions at the time of the crash. Most crashes occurred during daylight hours (76.4\%) and involved hitting another vehicle or being hit by another vehicle. Crashes occurred predominantly in urban areas (79.6\%).

Rollovers occurred in $2.6 \%$ of the crashes. Most crashes involved sedans (83.7\%, including station wagons) and SUV's (8.5\%). The mean travel speed upon impact was reported by police as 27.5 miles per hour (MPH), ranging from 0 to $97 \mathrm{MPH}$. Among the one-third of the cases that linked to a matching EMS run report, 16.4\% were reported to be in crashes with a change in velocity of greater than $20 \mathrm{MPH}$ and $44 \%$ received some kind of basic life support treatment while $15.1 \%$ received some type of advanced life support treatment.
Police reported $10 \%$ non-belt use and $16.6 \%$ unknown belt use status. In the subset of 1.878 cases that matched to an EMS report, reported non-belt use rate was 13.3\% (this included situations where only a lap belt or shoulder belt was worn). Airbags were reported by the police to have deployed in $17.1 \%$ of all crashes and by EMS personnel in $19.2 \%$ of the crashes linked to ambulance reports.

Suspected alcohol or drug involvement was reported by the police in $1.3 \%$ of the cases. Two-thirds of the drivers $(67.8 \%)$ were reported by the police to be transported to a medical facility though this does not necessarily mean transport by emergency medical services. Among the EMS cases, $80 \%$ were reportedly transported to a medical facility.

Approximately a third of the crashes occurred in each trimester; $35.0 \%$ the first trimester, $34.7 \%$ the second, and $30.3 \%$ the third trimester. Figure 2 shows the gestational age by week of the reported maternal crashes.

\section{DISCUSSION}

This study of motor vehicle crashes during pregnancy in Pennsylvania has some similarities and differences from the only other similar study that has been conducted. In a Utah linkage study for the period 1992 to 1999, it was reported that $2.8 \%$ of the live births experienced a maternal driver crash [11]. This is over twice as high as what was found in Pennsylvania. There are several possible reasons for this difference. It is useful to explore these reasons to understand how these factors might impact on other states experiences should they want to explore or conduct similar data linkages for surveillance purposes. 
From a demographic perspective, assuming for comparative purposes that environmental factors are about equal, maternal crash risk is driven in large part by the fertility of the underlying population (the number of births per year), the age distribution of the pregnant population, and the age-specific crash risk of the women of reproducetive age. For example, if the crash rate among young women is high but the mean age at pregnancy in that population occurs several years post peak crash risk, this population will have a lower maternal crash risk than a group with a similar rate and age distribution of crashes but a peak pregnancy pattern among younger mothers.

Figure 3 illustrates this by showing U.S. motor vehicle traffic injury rates (fatal and non-fatal) compared to birth rates, by age, for women ages 15 - 34. From a national perspective, it shows that fatal and non-fatal crash injury rates follow a similar age-specific pattern for women of reproductive age. They both rapidly increase at younger age groups from age 16 and peak at age 18, slowly declining over the next 12 years. With the age-specific birth rate overlaid on the crash injury pattern, Figure 3 shows that in the U.S. age-specific birth rates peak a little later than the crash risk. A different population (i.e., a sub-population such as a state or racial/ethnic group) that had the same age-specific pattern of crash risk but higher birth rates among younger women would probably have a higher rate of maternal crashes since crash injury rates are generally higher in younger women. So it is useful to observe that there appears to be a large variation among states in the fatal crash risk of women of reproductive age, ranging from 3.7 to 25.8 deaths per 100,000 women age 15 - 34 (see Figure 4) [12].

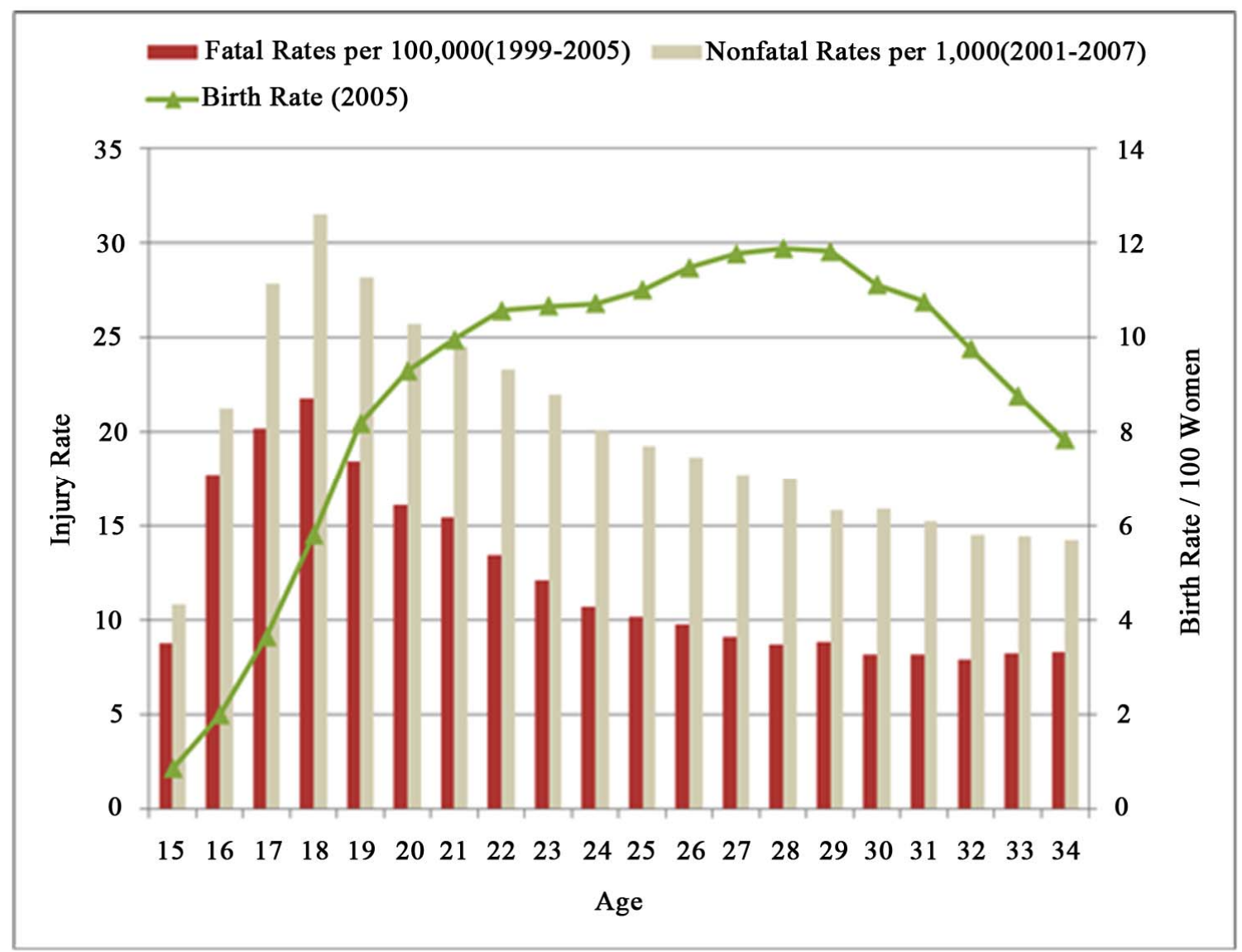

Figure 3. U.S. motor vehicle traffic injury rates (fatal and non-fatal) compared to birth rates, by age, for women ages 15 - 34. 'a. Fatal rates produced by the Centers for Disease Control and Prevention, National Center for Health Statistics. Compressed Mortality File 1999-2005). CDC WONDER On-line Database, compiled from Compressed Mortality File 1999-2005 Series 20 No. 2K, 2008. Accessed at http://wonder.cdc.gov/cmf-icd10.html on Jun 16, 2009. Non-fatal (emergency department visits) rates produced by WISQARS nonfatal injury reports from the office of statistics and programming, National Center for Injury Prevention and Control, CDC. URL: http://webappa.cdc.gov/s-asweb/ncipc/ nfirates2001.html. Data Source: NEISS all injury program operated by the Consumer Product Safety Commission for numbers of injuries. Bureau of Census for population estimates. Birth rates produced from the National Vital Statistics System Birth Tables, URL: http://www.cdc.gov/nchs/datawh/vitalstats/VtalStatsbirths.htm. Bureau of Census for population estimates. 


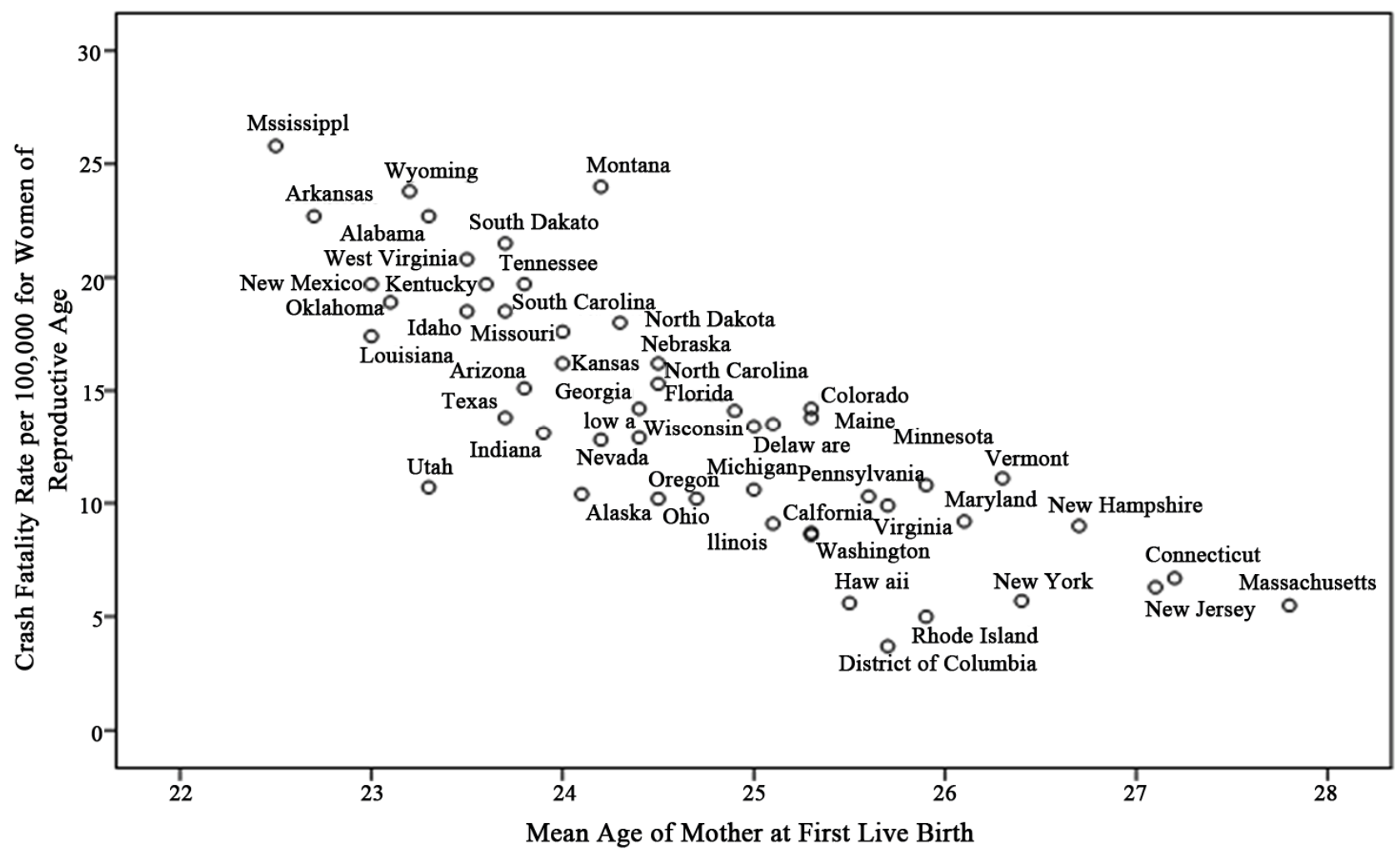

Figure 4. Scatter plot of mean age of mother at first live birth $(2000)^{\mathrm{a}}$ versus the crash fatality rate per 100,000 women of reproducetive age (15 - 34, 1999-2005), , by U.S. state.(a. Mean age of mother at first live birth by State (year 2000), from National Vital Statistics Reports, Vol. 51, No. 1, National Center for Health Statistics, 2002. (http://www.cdc.gov/nchs/data/nvsr/nvsr51/nvsr51_01.pdf). (b. Fatality data source: Centers for Disease Control and Prevention, National Center for Health Statistics. Compressed Mortality File 1999-2005). CDC WONDER On-line Database; 1999-2005 Series 20 No. 2K, 2008. Accessed at http://wonder.cdc.gov/cmf-icd10.htmlon Jun 16, 2009.

The only two states studied so far with crash and vital statistics linkage studies, Utah and Pennsylvania, have annualized vehicle traffic fatality rates for women of reproductive age of 10.7 and 10.3 per $1,000,000$ women, respectively for 1999-2005. But many states have twice this rate. As age-specific birth and crash rates act together, sub-populations can be expected to have very different rates of maternal crash involvement. In particular, states with both a high crash risk among young women of reproductive age and high birth rates among the same group of younger mothers would be at especially high risk of maternal/fetal crash injury.

Figure 4 shows a scatter plot of the mean age of mothers at first live birth by State for the year 2000 [13] with the motor vehicle traffic fatality rate per 100,000 women ages 15 - 34 for 1999-2005 (which as shown in Figure 2, correlates well with non-fatal crash injury rates) [11]. States in the upper left quadrant would be prime jurisdictions to explore whether they truly have elevated maternal crash injury rates. It also suggests that the rates of maternal crashes observed in Utah and Pennsylvania may be in the lower half of all the states and the lower rate observed in Pennsylvania compared to Utah may be due, in part, to the much younger mothers in Utah since younger women are at a higher risk of having a crash. It is also possible that successful linkage rates varied between Pennsylvania and Utah. Similar linking software and techniques were used, but differences in available variable types and state specific data collection procedures impacting on linkage quality and completeness cannot be ruled out.

Over one-third of the cases that linked to an EMS report were reported not to use the full lap/shoulder belt combination which parallels findings from the Utah study showing decreasing belt use as injury severity increased [11]. This level of misuse is of concern as the American College of Obstetricians and Gynecologists (ACOG) [14] and others [15] have long recommend the use of seatbelts during pregnancy and many studies have shown the benefits of seatbelt use [11,16,17]. Unfortunately, our study had no way to reliably ascertain proper belt use among the study population.

There were other limitations affecting case ascertainment. Fetal deaths before 20 week gestation are not reported to vital statistics and thus were not available for linkage. Pregnant women who may have been involved in a crash but moved out of state or gave birth in another state would also be missed. Excluding the second and 
third crashes for the small number with multiple crashes also underestimates the overall crash risk. Therefore, the incidence estimates probably err to the conservative side.

\section{CONCLUSIONS}

Using probabilistic linkage of crash and vital statistics records we determined that in Pennsylvania at least $1.1 \%$ of all pregnancies were involved in a driver related motor vehicle crash with little variance in risk over gestational age but higher crash rates among younger mothers. Pennsylvania's motor vehicle crash rate during pregnancy might be on the lower end of the risk spectrum compared to other states because of relatively older maternal ages at birth and the overall lower crash injury risk in older women.

These data, combined with other evidence [2], confirm that pregnancy related crashes occur at a higher rate than infant related crashes with a concomitant threat to the fetus and new-born that cannot be tracked within current crash data systems without on-going data linkage efforts. The National Highway Traffic Safety Administration (NHTSA) already has an existing network of more than a dozen state-based programs that currently link crash data to ambulance run reports called the Crash Outcome Data Evaluation System (CODES) [18]. It would not take many additional resources for those states to add linkages to state vital statistics (birth and fetal death) data, in effect setting up an inexpensive but robust pregnancy-related driver crash surveillance system for CODES states. Without such efforts this important threat to the fetus and newborn cannot be tracked and evaluated, remaining a hidden cause of crash injury and mortality to the very youngest of crash victims.

\section{ACKNOWLEDGEMENTS}

This work was supported by the Centers for Disease Control and Prevention, National Center for Injury Prevention and Control (grant CCR 323155) at the University of Pittsburgh, Department of Neurological Surgery, Center for Injury Research and Control. We gratefully acknowledge the assistance of Dr. Michael McGlincy of Strategic Matching Inc. who served as this study's honest broker and who performed all of the probabilistic linkages.

\section{REFERENCES}

[1] Rochat, R.W., et al. (1988) Maternal mortality in the United States: Report from the maternal mortality collaborative. Obstetrics Gynecology, 72, 91-97.

[2] Weiss, H.B. (2001) Causes of traumatic death during pregnancy (letter). Journal of the American Medical Association, 285, 2854-2855. doi:10.1001/jama.285.22.2854

[3] Weiss, H.B. (1999) Pregnancy-associated injury hospitalizations in Pennsylvania. Annals of Emergency Medi- cine, 34, 626-636. doi:10.1016/S0196-0644(99)70165-4

[4] Schiff, M.H.V.L. and Daling, J.R. (2001) Pregnancyassociated injury hospitalizations: Maternal and fetal outcomes. Paediatric and Perinatal Epidemiology, 15, A29. doi:10.1046/j.1365-3016.2001.00381-91.x

[5] Pearlman, M.D. (1997) Motor vehicle crashes, pregnancy loss and preterm labor. International Journal of Gynaecology and Obstetrics, 57, 127-132.

[6] Weiss, H.B. (2001) The epidemiology of traumatic injury-related fetal mortality in Pennsylvania, 1995-1997: The role of motor vehicle crashes. Accident Analysis and Prevention, 33, 449-454. doi:10.1016/S0001-4575(00)00058-0

[7] Centers for Disease Control and Prevention, National Center for Injury Prevention and Control. Web-Based Injury Statistics Query and Reporting System (WISQA RS) [online]. (2007) [cited 2011 Nov. 28]. http://www.cdc.gov/ncipc/wisqars

[8] Weiss, H.B. and Strotmeyer, S. (2002) Characteristics of pregnant women in motor vehicle crashes. Injury Prevention, 8, 207-210. doi:10.1136/ip.8.3.207

[9] U.S. Department of Transportation, National Highway Traffic Safety Administration. National Automotive Sampling System (NASS), 2008 General Estimates System (GES). [Web page] 2008 July 25, 2009]; Available from: ftp://ftp.nhtsa.dot.gov/GES/GES08/.

[10] Haapaniemi, P. (1996) Women's highway deaths on the rise. Traffic Safety, 96, 6-11.

[11] Hyde, L.K., et al. (2003) Effect of motor vehicle crashes on adverse fetal outcomes. Obstetrics Gynecology, 102, 279-286. doi:10.1016/S0029-7844(03)00518-0

[12] Centers for Disease Control and Prevention and National Center for Health Statistics. Compressed Mortality File 1999-2005. http://wonder.cdc.gov/cmf-icd10.html.

[13] Mathews, T.J. and Hamilton, B.E. (2002) Mean age of mother, 1970-2000. National Vital Statistics Reports, National Center for Health Statistics, 51, 1120-2003.

[14] American College of Obstetricians and Gynecologists (1991) Automobile passenger restraints for children and pregnant women. ACOG Technical Bulletin, 151.

[15] National Highway Traffic Safety Administration. SeatbeIts and Pregnancy Brochure, 2002; Available from: http://www.nhtsa.dot.gov/people/injury/airbags/Internet Sevices_Group/ISG-Restricted/Buckle-Up\%20America/ pregnancybrochure/BUA_PregnancyNHTSAchange.pdf.

[16] Manoogian, S.J., Moorcroft, D.M. and Duma, S.M (2008) Pregnant occupant injury risk in severe frontal crashes using computer simulations. Biomedical Sciences Instrumentation, 44, 249-255.

[17] Klinich, K.D., et al. (2008) Fetal outcome in motor-vehicle crashes: Effects of crash characteristics and maternal restraint. American Journal of Obstetrics and Gynecology, 198, E1-E9. doi:10.1016/j.ajog.2008.02.009

[18] Allen, M. and Weiss, H. (1998) Using linked data to evaluate child safety seat effectiveness in pennsylvania. Report from the Pennsylvania Crash Outcome Data Evaluation System to the National Highway Traffic Safety Administration. 